\title{
PBW theoretic approach to the module category of quantum affine algebras
}

\author{
By Masaki KashiwarA, M.J.A., ${ }^{* 1), * 2), * 3)}$ Myungho KIM, ${ }^{* 4)}$ Se-jin $\mathrm{OH}^{* 5)}$ and Euiyong PARK ${ }^{* 6)}$ \\ (Contributed by Masaki Kashiwara, M.J.A., May 12, 2021)
}

\begin{abstract}
Let $U_{q}^{\prime}(\mathfrak{g})$ be a quantum affine algebra of untwisted affine ADE type and let $\mathscr{C}_{\mathfrak{g}}^{0}$ be Hernandez-Leclerc's category. For a duality datum $\mathcal{D}$ in $\mathscr{C}_{\mathfrak{g}}^{0}$, we denote by $\mathcal{F}_{\mathcal{D}}$ the quantum affine Weyl-Schur duality functor. We give a sufficient condition for a duality datum $\mathcal{D}$ to provide the functor $\mathcal{F}_{\mathcal{D}}$ sending simple modules to simple modules. Moreover, under the same condition, the functor $\mathcal{F}_{\mathcal{D}}$ has compatibility with the new invariants introduced by the authors. Then we introduce the notion of cuspidal modules in $\mathscr{C}_{\mathfrak{g}}^{0}$, and show that all simple modules in $\mathscr{C}_{\mathfrak{g}}^{0}$ can be constructed as the heads of ordered tensor products of cuspidal modules. We next state that the ordered tensor products of cuspidal modules have the unitriangularity property.
\end{abstract}

Key words: Cuspidal modules; quantum affine Weyl-Schur duality; Hernandez-Leclerc category; quantum affine algebra; quiver Hecke algebra.

1. Introduction. Let $q$ be an indeterminate and let $\mathscr{C}_{\mathfrak{g}}$ be the category of finite-dimensional integrable modules over a quantum affine algebra $U_{q}^{\prime}(\mathfrak{g})$. The category $\mathscr{C}_{\mathfrak{g}}$ occupies an important position in the representation theory of quantum affine algebras because of its rich structure. The simple modules in $\mathscr{C}_{\mathfrak{g}}$ are parameterized using $n$-tuples of polynomials with constant term 1 (called Drinfeld polynomials), which was proved in [2-4] for the untwisted cases and in [5] for the twisted cases. Any simple module can be obtained as the head of ordered tensor product of fundamental modules, which was shown in $[1,13,27]$. A geometric approach to simple modules was also studied in [21,27].

Let $\mathfrak{g}_{0}$ be a finite-dimensional simple Lie algebra of ADE type and $\mathfrak{g}$ the untwisted affine

2010 Mathematics Subject Classification. Primary 17B37, 81R50; Secondary 18D10.

*1) Kyoto University Institute for Advanced Study, Yoshida Ushinomiya-cho, Sakyo-ku, Kyoto 606-8501, Japan.

*2) Research Institute for Mathematical Sciences, Kyoto University, Kitashirakawa-Oiwakecho, Sakyo-ku, Kyoto 6068502, Japan.

*3) Korea Institute for Advanced Study, 85 Hoegiro, Dongdaemun-gu, Seoul 02455, Republic of Korea.

*4) Department of Mathematics, Kyung Hee University, 26 Kyungheedae-ro, Dongdaemun-gu, Seoul 02447, Republic of Korea.

*5) Department of Mathematics, Ewha Womans University, 52 Ewhayeodae-gil, Seodaemun-gu, Seoul 03760, Republic of Korea.

*6) Department of Mathematics, University of Seoul, 163 Seoulsiripdae-ro, Dongdaemun-gu, Seoul 02504, Republic of Korea.
Lie algebra associated with $\mathfrak{g}_{0}$. Hernandez and Leclerc defined the full subcategory $\mathscr{C}_{\mathfrak{g}}^{0}$ of $\mathscr{C}_{\mathfrak{g}}$ such that all simple subquotients of its objects appear in tensor products of certain fundamental representations ([7]). Because any simple module in $\mathscr{C}_{\mathfrak{g}}$ can be obtained as a tensor product of suitable parameter shifts of simple modules in $\mathscr{C}_{\mathfrak{g}}^{0}$, the category $\mathscr{C}_{\mathfrak{g}}^{0}$ contains an essential information of $\mathscr{C}_{\mathfrak{g}}$. For each $Q$ data $\mathcal{Q}=(Q, \phi)$ of $\mathfrak{g}_{0}$, which is a pair of a Dynkin quiver $Q$ of $\mathfrak{g}_{0}$ and its height function $\phi$, Hernandez and Leclerc defined a monoidal subcategory $\mathscr{C}_{\mathcal{Q}}$ of $\mathscr{C}_{\mathfrak{g}}^{0}$ and proved that its complexified Grothendieck ring $\mathbf{C} \otimes_{\mathbf{z}} K\left(\mathscr{C}_{\mathcal{Q}}\right)$ is isomorphic to the coordinate ring $\mathbf{C}[N]$ of the unipotent group associated with $\mathfrak{g}_{0}$ ([8]). Under this isomorphism, the set of isomorphism classes of simple modules in $\mathscr{C}_{\mathcal{Q}}$ corresponds to the upper global base of $\mathbf{C}[N]$.

The quantum affine Weyl-Schur duality was introduced in [10]. This duality tells us that, for a duality datum $\mathcal{D}=\left\{L_{i}\right\}_{i \in J} \subset \mathscr{C}_{\mathfrak{g}}$, there exists a monoidal functor $\mathcal{F}_{\mathcal{D}}$ from the finite-dimensional graded module category $R$-gmod of the quiver Hecke algebra $R([19,25])$ determined by $\mathcal{D}$ to the category $\mathscr{C}_{\mathfrak{g}}$. In general, it is hard to find conditions for $\mathcal{D}$ to provide the functor $\mathcal{F}_{\mathcal{D}}$ with good properties. But, to each choice of $Q$-data $\mathcal{Q}$, we can associate a quantum affine Weyl-Schur duality functor $\mathcal{F}_{\mathcal{Q}}$ with good properties $([6,9])$ :

$$
\mathcal{F}_{\mathcal{Q}}: R_{\mathfrak{g}_{0}}-\operatorname{gmod} \longrightarrow \mathscr{C}_{\mathcal{Q}} \subset \mathscr{C}_{\mathfrak{g}}^{0},
$$

where $R_{\mathfrak{g}_{0}}$ is the symmetric quiver Hecke algebra associated with $\mathfrak{g}_{0}$. This functor $\mathcal{F}_{\mathcal{Q}}$ sends simple 
modules of $R_{\mathfrak{g}_{0}}$-gmod to simple modules of $\mathscr{C}_{\mathcal{Q}}$ and gives an isomorphism at the Grothendieck ring level.

One of the main results of this paper is to describe a sufficient condition for a duality datum $\mathcal{D}$ to provide the functor $\mathcal{F}_{\mathcal{D}}$ with good properties. Let $U_{q}^{\prime}(\mathfrak{g})$ be an arbitrary quantum affine algebra. Let $\mathcal{D}=\left\{L_{i}\right\}_{i \in J} \subset \mathscr{C}_{\mathfrak{g}}$ be a duality datum associated with a simply-laced finite Cartan matrix $C$ and let $R_{\mathrm{C}}$ be the symmetric quiver Hecke algebra associated with C. If $L_{i}$ are root modules (see (2.1)) and $\mathcal{D}$ satisfies condition (2.2) below, we say that $\mathcal{D}$ is a strong duality datum. We show that, for a strong duality datum $\mathcal{D}$, the duality functor $\mathcal{F}_{\mathcal{D}}$ sends simple modules to simple modules and it induces an injective ring homomorphism from $K_{q=1}\left(R_{\mathrm{C} \text {-gmod })}\right.$ to $K\left(\mathscr{C}_{\mathfrak{g}}\right)$ (see Theorem 2.1 ). Moreover, the duality functor is compatible with the new invariants:

$$
\begin{aligned}
& \Lambda(M, N)=\Lambda\left(\mathcal{F}_{\mathcal{D}}(M), \mathcal{F}_{\mathcal{D}}(N)\right), \\
& \mathfrak{d}(M, N)=\mathfrak{d}\left(\mathcal{F}_{\mathcal{D}}(M), \mathcal{F}_{\mathcal{D}}(N)\right), \\
& (\operatorname{wt}(M), \operatorname{wt}(N))=-\Lambda^{\infty}\left(\mathcal{F}_{\mathcal{D}}(M), \mathcal{F}_{\mathcal{D}}(N)\right)
\end{aligned}
$$

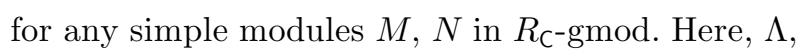
$\mathfrak{d}$ and $\Lambda^{\infty}$ are new invariants for pairs of objects of $\mathscr{C}_{\mathfrak{g}}$ introduced in [14]. These invariants are quantum affine algebra analogues of the invariants (with the same notations) for the quiver Hecke algebras (see $[10,12])$. Note that the block decompositions for $\mathscr{C}_{\mathfrak{g}}$ and $\mathscr{C}_{\mathfrak{g}}^{0}$ were given by using the new invariant $\Lambda^{\infty}$ in $[15]$.

The other main theorem of this paper is to construct all simple modules in $\mathscr{C}_{\mathfrak{g}}^{0}$ as the heads of ordered tensor products of cuspidal modules, which can be understood as a generalization of the simple module construction using ordered tensor products of fundamental modules $([4,22])$.

Suppose that $\mathfrak{g}_{0}$ is a finite-dimensional simple Lie algebra of $A D E$ type and $\mathfrak{g}$ is the untwisted affine Lie algebra associated with $\mathfrak{g}_{0}$. Let $\mathcal{Q}$ be a $Q$-data of $\mathfrak{g}_{0}$ and let $\mathcal{D}_{\mathcal{Q}}$ be the duality datum induced by $\mathcal{Q}$. Let $\mathcal{F}_{\mathcal{Q}}:=\mathcal{F}_{\mathcal{D}_{\mathcal{Q}}}$ be the duality functor associated with $\mathcal{D}_{\mathcal{Q}}$. Since $\mathcal{D}_{\mathcal{Q}}$ is a strong duality datum, $\mathcal{F}_{\mathcal{Q}}$ sends simples to simples and preserves the new invariants, i.e., (1.1) holds.

Let $w_{0}$ be the longest element of the Weyl group $W_{0}$ of $\mathfrak{g}_{0}$, and $\ell$ the length of $w_{0}$. We choose an arbitrary reduced expression $w_{0}$ of $w_{0}$. We define the cuspidal modules $\left\{\mathrm{S}_{k}\right\}_{k \in \mathbf{Z}} \subset \overline{\mathscr{C}_{\mathfrak{g}}^{0}}$ to be the simple $U_{q}^{\prime}(\mathfrak{g})$-modules given by

(a) $\mathrm{S}_{k}=\mathcal{F}_{\mathcal{Q}}\left(V_{k}\right)$ for any $k=1, \ldots, \ell$, (b) $\mathrm{S}_{k+\ell}=\mathscr{D}\left(\mathrm{S}_{k}\right)$ for any $k \in \mathbf{Z}$,

where $\left\{V_{k}\right\}_{k=1, \ldots, \ell} \subset R_{\mathfrak{g}_{0}}$-gmod are the cuspidal modules associated with $\underline{w_{0}}$, and $\mathscr{D}(X)$ and $\mathscr{D}^{-1}(X)$ denote the right dual and the left dual of a module $X \in \mathscr{C}_{\mathfrak{g}}$, respectively. Note that the cuspidal module $V_{k}$ corresponds to the dual $P B W$ vectors associated with $\underline{w_{0}}$ under the categorification using quiver Hecke algebras.

For any $\mathbf{a}=\left(a_{k}\right)_{k \in \mathbf{Z}} \in \mathbf{Z}:=\mathbf{Z}_{\geq 0}^{\oplus \mathbf{Z}}$, we define the ordered tensor product by

$$
\mathrm{P}_{Q, \underline{w_{0}}}(\mathbf{a}):=\cdots \otimes \mathrm{S}_{1}^{\otimes a_{1}} \otimes \mathrm{S}_{0}^{\otimes a_{0}} \otimes \mathrm{S}_{-1}^{\otimes a_{-1}} \otimes \cdots
$$

We prove that the head hd( $\left.\mathrm{P}_{Q, w_{0}}(\mathbf{a})\right)$ of the ordered tensor product $\mathrm{P}_{Q, w_{0}}(\mathbf{a})$ is simple and the simple module $\mathrm{hd}\left(\mathrm{P}_{Q, w_{0}}(\mathbf{a})\right)$ appears only once in $\mathrm{P}_{Q, w_{0}}(\mathbf{a})$. Moreover, for any simple module $V \in \mathscr{C}_{\mathfrak{g}}^{0}$, there exists a unique $\mathbf{a} \in \mathbf{Z}$ such that $V$ is isomorphic to the head $\operatorname{hd}\left(\mathrm{P}_{Q, w_{0}}(\mathbf{a})\right)$. Such an $\mathbf{a}$ is denoted by $\mathbf{a}_{Q, w_{0}}(V)$.

Thus, setting $\mathrm{V}_{Q, w_{0}}(\mathbf{a}):=\operatorname{hd}\left(\mathrm{P}_{Q, w_{0}}(\mathbf{a})\right)$, the set $\left\{\mathrm{V}_{Q, w_{0}}(\mathbf{a}) \mid \mathbf{a} \in \mathbf{Z}\right\}$ is a complete and irredundant set of simple modules of $\mathscr{C}_{\mathfrak{g}}^{0}$ up to isomorphisms (see Theorem 3.4). We prove further that, if $V$ is a simple subquotient of $\mathrm{P}_{Q, \underline{w_{0}}}(\mathbf{a})$ which is not isomorphic to $\mathrm{V}_{Q, \underline{w_{0}}}(\mathbf{a})$, then

$$
\mathbf{a}_{Q, \underline{w_{0}}}(V) \prec \mathbf{a},
$$

where $\prec$ is the bi-lexicographic order on $\mathbf{Z}$ (see $(3.2))$. Thus the family of modules $\left\{\mathrm{P}_{Q, w_{0}}(\mathbf{a})\right\}_{\mathbf{a} \in \mathbf{Z}}$ has the unitriangularity property with respect to $\prec$ (see Theorem 3.5).

We can generalize the above results to an arbitrary quantum affine algebra $U_{q}^{\prime}(\mathfrak{g})$ including twisted type by using certain strong duality datum in $\mathscr{C}_{\mathfrak{g}}$ (see Remark 3.6).

This paper is an announcement whose details will appear elsewhere.

2. Quantum affine Weyl-Schur duality. Let $\mathbf{k}$ be the algebraic closure of the subfield $\mathbf{Q}(q)$ in the algebraically closed field $\bigcup_{m>0} \mathbf{C}\left(\left(q^{1 / m}\right)\right)$. Let $U_{q}^{\prime}(\mathfrak{g})$ be the quantum affine algebra over the base field $\mathbf{k}$ associated with an affine Cartan matrix $\mathrm{A}=$ $\left(a_{i, j}\right)_{i, j \in I}$, and set $\mathscr{C}_{\mathfrak{g}}$ to be the category of finitedimensional integrable $U_{q}^{\prime}(\mathfrak{g})$-modules.

Let $\mathbf{C}=\left(c_{i, j}\right)_{i, j \in J}$ be a simply-laced finite Cartan matrix and $R_{\mathrm{C}}$ the symmetric quiver Hecke algebra associated with $\mathrm{C}$. We denote by $K\left(R_{\mathrm{C}}\right.$-gmod $)$ the Grothendieck ring of the category

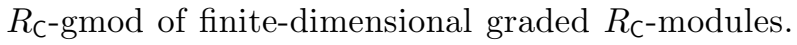
Note that $K\left(R_{\mathrm{C}}\right.$-gmod $)$ is isomorphic to the quan- 
tum unipotent coordinate ring $A_{q}(\mathrm{C})_{\mathbf{Z}\left[q, q^{-1}\right]}([19,25])$ and the set of isomorphism classes of simple $R_{\mathrm{C}}$-modules corresponds to the upper global basis

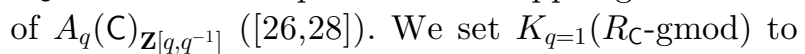
be the specialization of $K\left(R_{\mathrm{C}}\right.$-gmod $)$ at $q=1$.

We freely use new invariants $\Lambda, \Lambda^{\infty}$ and $\mathfrak{d}$ for pairs of modules in $\mathscr{C}_{\mathfrak{g}}$ introduced in [14].

A simple $U_{q}^{\prime}(\mathfrak{g})$-module $M$ is real if $M \otimes M$ is simple. A root module is a real simple module $L$ such that

$$
\mathfrak{d}\left(L, \mathscr{D}^{k}(L)\right)=\delta(k= \pm 1) \quad \text { for any } k \in \mathbf{Z} .
$$

Let $\mathcal{D}:=\left\{L_{i}\right\}_{i \in J} \subset \mathscr{C}_{\mathfrak{g}}$ be a family of simple modules of $\mathscr{C}_{\mathfrak{g}}$. The family $\mathcal{D}$ is called a duality datum associated with $C$ if it satisfies the following

(a) for each $i \in J, L_{i}$ is a real simple module,

(b) for any $i, j \in J$ with $i \neq j, \mathfrak{d}\left(L_{i}, L_{j}\right)=-c_{i, j}$. Then one can construct a monoidal functor

$$
\mathcal{F}_{\mathcal{D}}: R_{C^{-} \operatorname{gmod}} \longrightarrow \mathscr{C}_{\mathfrak{g}}
$$

using the duality datum $\mathcal{D}$ (see $[10,17]$ ). Moreover, $\mathcal{F}_{\mathcal{D}}$ is an exact functor. The functor $\mathcal{F}_{\mathcal{D}}$ is called a quantum affine Weyl-Schur duality or shortly a duality functor.

A strong duality datum is a duality datum $\mathcal{D}=$ $\left\{L_{i}\right\}_{i \in J}$ such that all $L_{i}$ are root modules and

$$
\mathfrak{d}\left(L_{i}, \mathscr{D}^{k}\left(L_{j}\right)\right)=-\delta(k=0) c_{i, j}
$$

for any $k \in \mathbf{Z}$ and $i, j \in J$ such that $i \neq j$.

Theorem 2.1. Let $\mathcal{D}=\left\{L_{i}\right\}_{i \in J}$ be a strong duality datum associated with a simply-laced finite Cartan matrix $\mathrm{C}=\left(c_{i, j}\right)_{i, j \in J}$. Then we obtain the following

(i) The duality functor $\mathcal{F}_{\mathcal{D}}$ sends simple modules to simple modules.

(ii) The duality functor $\mathcal{F}_{\mathcal{D}}$ induces an injective ring homomorphism from $K_{q=1}\left(R_{\left.C^{-} \text {gmod }\right)}\right.$ to $K\left(\mathscr{C}_{\mathfrak{g}}\right)$.

The duality functor also has compatibility with the new invariants.

Theorem 2.2. Let $\mathcal{D}=\left\{L_{i}\right\}_{i \in J}$ be a strong duality datum. Then, for any simple modules $M, N$ in $R_{\mathrm{C}}$-gmod, we have

(i) $\Lambda(M, N)=\Lambda\left(\mathcal{F}_{\mathcal{D}}(M), \mathcal{F}_{\mathcal{D}}(N)\right)$,

(ii) $\mathfrak{d}(M, N)=\mathfrak{d}\left(\mathcal{F}_{\mathcal{D}}(M), \mathcal{F}_{\mathcal{D}}(N)\right)$,

(iii) $($ wt $M$, wt $N)=-\Lambda^{\infty}\left(\mathcal{F}_{\mathcal{D}}(M), \mathcal{F}_{\mathcal{D}}(N)\right)$,

(iv) $\mathfrak{d}\left(\mathscr{D}^{k} \mathcal{F}_{\mathcal{D}}(M), \mathcal{F}_{\mathcal{D}}(N)\right)=0$ for any $k \neq 0, \pm 1$,

(v) $\widetilde{\Lambda}(M, N)=\mathfrak{d}\left(\mathscr{D} \mathcal{F}_{\mathcal{D}}(M), \mathcal{F}_{\mathcal{D}}(N)\right)$

$$
=\mathfrak{d}\left(\mathcal{F}_{\mathcal{D}}(M), \mathscr{D}^{-1} \mathcal{F}_{\mathcal{D}}(N)\right) .
$$

3. PBW theoretic approach. Let $\mathfrak{g}_{0}$ be a finite-dimensional simple Lie algebra of ADE type, and $\mathfrak{g}$ the untwisted affine Kac-Moody algebra associated with $\mathfrak{g}_{0}$. We denote by $\mathrm{A}=\left(a_{i, j}\right)_{i, j \in I}$ the affine Cartan matrix of $\mathfrak{g}$ and by $I_{0} \subset I$ the index set corresponding to $\mathfrak{g}_{0}$. For each $i \in I_{0}$ and $c \in \mathbf{k}^{\times}$, let $V\left(\varpi_{i}\right)_{c}$ be the $i$-th fundamental representation.

Following [7], we denote by $\mathscr{C}_{\mathfrak{g}}^{0}$ the smallest full subcategory of the category $\mathscr{C}_{\mathfrak{g}}$ such that

(a) it contains $\left\{V\left(\varpi_{i}\right)_{(-q)^{p}} \mid i \in I_{0}, p \equiv d(1, i)\right.$ $\bmod 2\}$,

(b) it is stable under taking subquotients, extensions, and tensor products.

Here 1 is an arbitrarily chosen element of $I_{0}$ and $d(1, i)$ is the distance between 1 and $i$ in the Dynkin diagram of $\mathfrak{g}_{0}$.

A $Q$-data $\mathcal{Q}=\left(Q, \phi_{Q}\right)$ is a pair of a Dynkin quiver $Q$ of $\mathfrak{g}_{0}$ and a function $\phi_{Q}(i): I_{0} \rightarrow \mathbf{Z}$ such that $\phi_{Q}(1) \in 2 \mathbf{Z}$ and $\phi_{Q}(i)=\phi_{Q}(j)+1$ for any arrow $i \rightarrow j$ in $Q$. The function $\phi_{Q}$ is called a height function of $Q$. For any $Q$-data $\mathcal{Q}$, we denote by $\mathscr{C}_{\mathcal{Q}}$ the full monoidal subcategory of $\mathscr{C}_{\mathfrak{g}}^{0}$ introduced in [8]. For $i \in I_{0}$, let $L_{i}$ be the fundamental representation in $\mathscr{C}_{\mathcal{Q}}$ corresponding to the simple root $\alpha_{i}$ in the Auslander-Reiten quiver of $\mathcal{Q}$. Then $\left\{L_{i}\right\}_{i \in I_{0}}$ forms a strong duality datum and it induces a duality functor

$$
\mathcal{F}_{\mathcal{Q}}: R_{\mathfrak{g}_{0}} \text {-gmod } \longrightarrow \mathscr{C}_{\mathcal{Q}} \subset \mathscr{C}_{\mathfrak{g}}^{0}
$$

$([6,9])$. Here $R_{\mathfrak{g}_{0}}$ is the symmetric quiver Hecke algebra associated with $\mathfrak{g}_{0}$. Note that the functor $\mathcal{F}_{\mathcal{Q}}$ is an equivalence of categories between $R_{\mathfrak{g}_{0}}$-gmod and $\mathscr{C}_{\mathcal{Q}}$ after forgetting grading ([6]).

Hence Theorem 2.2 implies the following result.

Theorem 3.1. Let $\mathcal{Q}$ be a Q-data of $\mathfrak{g}_{0}$. For any simple modules $M$ and $N$ in $R_{\mathfrak{g}_{0}}$-gmod, we have

(i) $\Lambda(M, N)=\Lambda\left(\mathcal{F}_{\mathcal{Q}}(M), \mathcal{F}_{\mathcal{Q}}(N)\right)$,

(ii) $(\operatorname{wt}(M), \operatorname{wt}(N))=-\Lambda^{\infty}\left(\mathcal{F}_{\mathcal{Q}}(M), \mathcal{F}_{\mathcal{Q}}(N)\right)$.

Let $\Delta_{0}^{+}$be the set of positive roots of $\mathfrak{g}_{0}$ and let $\mathrm{W}_{0}=\left\langle r_{i} \mid i \in I_{0}\right\rangle$ be the Weyl group associated with $\mathfrak{g}_{0}$, where $r_{i}$ is the $i$-th reflection. Let $w_{0}$ be the longest element of $\mathrm{W}_{0}$, and $\ell$ denotes the length of $w_{0}$. We choose an arbitrary reduced expression $w_{0}=r_{i_{1}} r_{i_{2}} \cdots r_{i_{\ell}}$ of the longest element $w_{0}$ of $W_{0}$. (We do not assume that $\underline{w_{0}}$ is $Q$-adapted.) Then we have $\Delta_{0}^{+}=\left\{\beta_{k} \mid k=1, \ldots, \ell\right\}$, where

$$
\beta_{k}:=r_{i_{1}} \cdots r_{i_{k-1}}\left(\alpha_{i_{k}}\right) \text { for } k=1, \ldots, \ell .
$$

The reduced expression $\underline{w_{0}}$ gives the convex order 
on $\Delta_{0}^{+}$defined by $\beta_{a}>\beta_{b}$ for any $a>b$, and provides the PBW vectors $\left\{E\left(\beta_{k}\right)\right\}_{k=1, \ldots, \ell}$ of the negative half $U_{\mathbf{Z}\left[q, q^{-1}\right]}^{-}\left(\mathfrak{g}_{0}\right)$ and its dual vectors $\left\{E^{*}\left(\beta_{k}\right)\right\}_{k=1, \ldots, \ell}$ of the quantum unipotent coordinate ring $A_{q}\left(\mathfrak{n}_{0}\right)_{\mathbf{Z}\left[q, q^{-1}\right]}$, where $\mathfrak{n}_{0}$ is the maximal nilpotent subalgebra of $\mathfrak{g}_{0}$.

Let $\left\{V_{k}\right\}_{k=1, \ldots, \ell} \subset R_{\mathfrak{g}_{0}}$-gmod be the cuspidal modules associated with the reduced expression $w_{0}([18,20])$. Under the isomorphism between $\bar{K}\left(R_{\mathfrak{g}_{0}}\right.$-gmod $)$ and $A_{q}\left(\mathfrak{n}_{0}\right)_{\mathbf{Z}\left[q, q^{-1}\right]}$, the cuspidal module $V_{k}$ corresponds to the dual PBW vector $E^{*}\left(\beta_{k}\right)$ for $k=1, \ldots, \ell$. It is known that the modules

$$
\left\{\operatorname{hd}\left(V_{\ell}^{\circ a_{\ell}} \circ \cdots \circ V_{1}^{\circ a_{1}}\right) \mid\left(a_{1}, \ldots, a_{\ell}\right) \in \mathbf{Z}_{\geq 0}^{\ell}\right\}
$$

gives a complete set of pairwise non-isomorphic simple graded $R_{\mathfrak{g}_{0}}$-modules $([18,20])$. Here $\circ$ denotes the convolution product in $R_{\mathfrak{g}_{0}}$-gmod.

We now introduce the notion of cuspidal modules for quantum affine algebras.

Definition 3.2. We define a sequence of simple $U_{q}^{\prime}(\mathfrak{g})$-modules $\left\{\mathrm{S}_{k}\right\}_{k \in \mathbf{Z}} \subset \mathscr{C}_{\mathfrak{g}}^{0}$ as follows:

(a) $\mathrm{S}_{k}=\mathcal{F}_{\mathcal{Q}}\left(V_{k}\right)$ for any $k=1, \ldots, \ell$, and we extend its definition to all $k \in \mathbf{Z}$ by

(b) $\mathrm{S}_{k+\ell}=\mathscr{D}\left(\mathrm{S}_{k}\right)$ for any $k \in \mathbf{Z}$.

The modules $\mathrm{S}_{k}(k \in \mathbf{Z})$ are called the cuspidal modules corresponding to the $Q$-data $\mathcal{Q}$ and the reduced expression $w_{0}$.

It is known that the cuspidal modules are fundamental representations if $w_{0}$ is $Q$-adapted.

However, they may not be fundamental in general.

We have the following property.

\section{Proposition 3.3.}

(i) $\mathrm{S}_{k}$ is a root module for any $k \in \mathbf{Z}$, i.e., $\mathrm{S}_{k}$ is a real simple module satisfying (2.1).

(ii) $\mathfrak{d}\left(\mathrm{S}_{j}, \mathscr{D}\left(\mathrm{S}_{k}\right)\right)=0$ for any $j<k$.

We define

$$
\mathbf{Z}:=\mathbf{Z}_{\geq 0}^{\oplus \mathbf{Z}}=\left\{\left(a_{k}\right)_{k \in \mathbf{Z}} \in \mathbf{Z}_{\geq 0}^{\mathbf{Z}} \mid \sum_{k \in \mathbf{Z}} a_{k}<\infty\right\} .
$$

We denote by $\prec$ the bi-lexicographic order on $\mathbf{Z}$, i.e., for any $\mathbf{a}=\left(a_{k}\right)_{k \in \mathbf{Z}}$ and $\mathbf{a}^{\prime}=\left(a_{k}^{\prime}\right)_{k \in Z}$ in $\mathbf{Z}, \mathbf{a} \prec \mathbf{a}^{\prime}$ if and only if the following conditions hold:

$$
\left\{\begin{array}{l}
\text { (a) there exists } r \in \mathbf{Z} \text { such that } a_{k}=a_{k}^{\prime} \\
\text { for any } k<r \text { and } a_{r}<a_{r}^{\prime}, \\
\text { (b) there exists } s \in \mathbf{Z} \text { such that } a_{k}=a_{k}^{\prime} \\
\text { for any } k>s \text { and } a_{s}<a_{s}^{\prime} .
\end{array}\right.
$$

For $\mathbf{a}=\left(a_{k}\right)_{k \in \mathbf{Z}} \in \mathbf{Z}$, we define

$$
\begin{aligned}
\mathrm{P}_{Q, \underline{w_{0}}}(\mathbf{a}) & :=\bigotimes_{k=+\infty}^{-\infty} \mathrm{S}_{k}^{\otimes a_{k}} \\
& =\cdots \otimes \mathrm{S}_{2}^{\otimes a_{2}} \otimes \mathrm{S}_{1}^{\otimes a_{1}} \otimes \mathrm{S}_{0}^{\otimes a_{0}} \otimes \mathrm{S}_{-1}^{\otimes a_{-1}} \otimes \cdots
\end{aligned}
$$

Here $\mathrm{P}_{Q, w_{0}}(0)$ should be understood as the trivial module $\overline{\mathbf{1}}$. We call the modules $\mathrm{P}_{Q, w_{0}}(\mathbf{a})$ standard modules with respect to the cuspidal modules $\left\{\mathrm{S}_{k}\right\}_{k \in \mathbf{Z}}$.

\section{Theorem 3.4.}

(i) For any $\mathbf{a} \in \mathbf{Z}$, the head of $\mathrm{P}_{Q, w_{0}}(\mathbf{a})$ is simple. We denote the head by

$$
\mathrm{V}_{Q, \underline{w_{0}}}(\mathbf{a}):=\operatorname{hd}\left(\mathrm{P}_{Q, \underline{w_{0}}}(\mathbf{a})\right) .
$$

(ii) For any simple module $V \in \mathscr{C}_{\mathfrak{g}}^{0}$, there exists a unique $\mathbf{a} \in \mathbf{Z}$ such that

$$
V \simeq \mathrm{V}_{Q, \underline{w_{0}}}(\mathbf{a}) .
$$

Therefore the set $\left\{\mathrm{V}_{Q, w_{0}}(\mathbf{a}) \mid \mathbf{a} \in \mathbf{Z}\right\}$ is a complete and irredundant set of simple modules of $\mathscr{C}_{\mathfrak{g}}^{0}$ up to isomorphisms.

Indeed, (i) follows from Proposition 3.3 ([14]).

The element $\mathbf{a} \in \mathbf{Z}$ associated with a simple module $V$ in Theorem 3.4 (ii) is called the cuspidal decomposition of $V$ with respect to the cuspidal modules $\left\{\mathrm{S}_{k}\right\}_{k \in \mathbf{Z}}$, and it is denoted by

$$
\mathbf{a}_{Q, \underline{w_{0}}}(V):=\mathbf{a} .
$$

Theorem 3.5. Let $\mathbf{a}$ be an element of $\mathbf{Z}$. Then we have the following

(i) The simple module $\mathrm{V}_{Q, w_{0}}(\mathbf{a})$ appears only once in $\mathrm{P}_{Q, w_{0}}(\mathbf{a})$.

(ii) If $V$ is a simple subquotient of $\mathrm{P}_{Q, w_{0}}(\mathbf{a})$ which is not isomorphic to $\mathrm{V}_{Q, w_{0}}(\mathbf{a})$, then we have

$$
\mathbf{a}_{Q, w_{0}}(V) \prec \mathbf{a} \text {. }
$$

(iii) In the Grothendieck ring, we have

$$
\left[\mathrm{P}_{Q, \underline{w_{0}}}(\mathbf{a})\right]=\left[\mathrm{V}_{Q, \underline{w_{0}}}(\mathbf{a})\right]+\sum_{\mathbf{a}^{\prime} \prec \mathbf{a}} c\left(\mathbf{a}^{\prime}\right)\left[\mathrm{V}_{Q, \underline{w_{0}}}\left(\mathbf{a}^{\prime}\right)\right],
$$

for some $c\left(\mathbf{a}^{\prime}\right) \in \mathbf{Z}_{\geq 0}$.

Remark 3.6. In $[11,16,23,24]$, the categories $\mathscr{C}_{\mathcal{Q}} \subset \mathscr{C}_{\mathfrak{g}}^{0}$ for untwisted non simply-laced affine types and twisted affine types are introduced, and a strong duality datum $\mathcal{D}=\left\{L_{i}\right\}_{i \in J}$ for each $\mathscr{C}_{\mathcal{Q}}$ is given. Then we can obtain the duality functor

$$
\mathcal{F}_{\mathcal{D}}: R_{\mathrm{C}-\operatorname{gmod}} \longrightarrow \mathscr{C}_{\mathcal{Q}} \subset \mathscr{C}_{\mathfrak{g}}^{0},
$$

where $\mathrm{C}$ denotes the simply-laced finite Cartan matrix determined by the affine type of $\mathfrak{g}$ (cf. [15]). 
Using the functors $\mathcal{F}_{\mathcal{D}}$, we can extend all the results in Section 3 to an arbitrary quantum affine algebra with an arbitrary choice of $w_{0}$ in type of C.

Acknowledgements. The research of Kashiwara was supported by Grant-in-Aid for Scientific Research (B) 15H03608, Japan Society for the Promotion of Science, the research of Kim was supported by the National Research Foundation of Korea (NRF) Grant funded by the Korea government (MSIP) (NRF-2017R1C1B2007824) and the research of Oh was supported by the Ministry of Education of the Republic of Korea and the NRF of Korea (NRF-2019R1A2C4069647).

\section{References}

[ 1 ] T. Akasaka and M. Kashiwara, Finite-dimensional representations of quantum affine algebras, Publ. Res. Inst. Math. Sci. 33 (1997), no. 5, 839-867.

[ 2 ] V. Chari and A. Pressley, Quantum affine algebras, Comm. Math. Phys. 142 (1991), no. 2, 261-283.

[ 3 ] V. Chari and A. Pressley, A guide to quantum groups, Cambridge University Press, Cambridge, 1994.

[ 4 ] V. Chari and A. Pressley, Quantum affine algebras and their representations, in Representations of groups (Banff, AB, 1994), 59-78, CMS Conf. Proc., 16, Amer. Math. Soc., Providence, RI, 1995.

[ 5 ] V. Chari and A. Pressley, Twisted quantum affine algebras, Comm. Math. Phys. 196 (1998), no. $2,461-476$.

[ 6 ] R. Fujita, Geometric realization of Dynkin quiver type quantum affine Schur-Weyl duality, Int. Math. Res. Not. IMRN 2020, no. 22, 83538386 .

[ 7 ] D. Hernandez and B. Leclerc, Cluster algebras and quantum affine algebras, Duke Math. J. 154 (2010), no. 2, 265-341.

[ 8 ] D. Hernandez and B. Leclerc, Quantum Grothendieck rings and derived Hall algebras, J. Reine Angew. Math. 701 (2015), 77-126.

[ 9 ] S.-J. Kang, M. Kashiwara and M. Kim, Symmetric quiver Hecke algebras and $R$-matrices of quantum affine algebras, II, Duke Math. J. 164 (2015), no. 8, 1549-1602.

[ 10 ] S.-J. Kang, M. Kashiwara and M. Kim, Symmetric quiver Hecke algebras and $R$-matrices of quantum affine algebras, Invent. Math. 211 (2018), no. 2, 591-685.

[11 ] S.-J. Kang, M. Kashiwara, M. Kim and S.-j. Oh, Symmetric quiver Hecke algebras and $R$-matrices of quantum affine algebras IV, Selecta
Math. (N.S.) 22 (2016), no. 4, 1987-2015.

[ 12 ] S.-J. Kang, M. Kashiwara, M. Kim and S.-j. Oh, Monoidal categorification of cluster algebras, J. Amer. Math. Soc. 31 (2018), no. 2, 349-426.

[13 ] M. Kashiwara, On level-zero representations of quantized affine algebras, Duke Math. J. 112 (2002), no. 1, 117-175.

[14] M. Kashiwara, M. Kim, S.-j. Oh and E. Park, Monoidal categorification and quantum affine algebras, Compos. Math. 156 (2020), no. 5, 1039-1077.

[15] M. Kashiwara, M. Kim, S.-j. Oh and E. Park, Simply-laced root systems arising from quantum affine algebras, arXiv:2003.03265v2.

[ 16 ] M. Kashiwara and S.-j. Oh, Categorical relations between Langlands dual quantum affine algebras: doubly laced types, J. Algebraic Combin. 49 (2019), no. 4, 401-435.

[17 ] M. Kashiwara and E. Park, Affinizations and $R$-matrices for quiver Hecke algebras, J. Eur. Math. Soc. (JEMS) 20 (2018), no. 5, 1161-1193.

[18 ] S. Kato, Poincaré-Birkhoff-Witt bases and Khovanov-Lauda-Rouquier algebras, Duke Math. J. 163 (2014), no. 3, 619-663.

[ 19 ] M. Khovanov and A. D. Lauda, A diagrammatic approach to categorification of quantum groups. I, Represent. Theory 13 (2009), 309347.

[20 ] P. J. McNamara, Finite dimensional representations of Khovanov-Lauda-Rouquier algebras I: Finite type, J. Reine Angew. Math. 707 (2015), 103-124.

[21] H. Nakajima, Quiver varieties and finite-dimensional representations of quantum affine algebras, J. Amer. Math. Soc. 14 (2001), no. 1, 145238.

[22] H. Nakajima, Quiver varieties and $t$-analogs of $q$-characters of quantum affine algebras, Ann. of Math. (2) 160 (2004), no. 3, 1057-1097.

[ 23 ] S.-j. Oh and T. Scrimshaw, Categorical relations between Langlands dual quantum affine algebras: exceptional cases, Comm. Math. Phys. 368 (2019), no. 1, 295-367.

[24] U. R. Suh and S.-j. Oh, Twisted and folded Auslander-Reiten quivers and applications to the representation theory of quantum affine algebras, J. Algebra 535 (2019), 53-132.

[ 25 ] R. Rouquier, 2-Kac-Moody algebras, arXiv:0812. $5023 \mathrm{v} 1$.

[26] R. Rouquier, Quiver Hecke algebras and 2-Lie algebras, Algebra Colloq. 19 (2012), no. 2, 359410.

[ 27 ] M. Varagnolo and E. Vasserot, Standard modules of quantum affine algebras, Duke Math. J. 111 (2002), no. 3, 509-533.

[28 ] M. Varagnolo and E. Vasserot, Canonical bases and KLR-algebras, J. Reine Angew. Math. 659 (2011), 67-100. 